\title{
Comparison of Fixed and Mobile Wimax Systems using hybrid DSI-PTS Technique for PAPR Reduction
}

\author{
Gurpriya Sandhu \\ M.E. Student \\ Department of Electronics and Communication \\ Engineering \\ National Institute Technical Teachers' Training and \\ Research, Sec. 26 Chandigarh
}

\author{
Garima Saini \\ Assistant Professor \\ Department of Electronics and Communication \\ Engineering \\ National Institute of Technical Teachers' Training \\ and Research, Sec. 26 Chandigarh
}

\begin{abstract}
Partial Transmit Sequence and Dummy Sequence Insertion Techniques are well-known distortionless Peak to Average Power Ratio Reduction (PAPR) techniques proposed to improve performance of Orthogonal Frequency Division Multiplexing (OFDM) Systems. In this paper, to improve the performance in terms of Peak to Average Power Ratio, Partial Transmit Sequence (PTS) technique has been proposed. Peak to average power ratio is the major issue of concern in OFDM system which reduces the efficiency of the high power amplifier. The computational complexity of conventional Partial Transmit Sequence is greater than the DSI-PTS technique and the PAPR results are outperformed by the latter.. A combination of Dummy Sequence Insertion technique and Partial Transmit Sequence has been considered for PAPR and complexity reduction. This technique has been simulated for scalable OFDM system. The simulation and results are presented for IEEE 802.16e standard.
\end{abstract}

\section{Keywords}

Partial Transmit Sequence (PTS), Orthogonal Frequency Division Multiplexing (OFDM), Dummy Sequence Insertion (DSI), Peak to Average Power Ratio (PAPR).

\section{INTRODUCTION}

OFDM has become relatively popular and accepted for its attractive features such as its robustness over frequency selective fading channels and high bandwidth efficiency. Despite its benefits, it suffers from some drawbacks such as Peak to Average Power Ratio in the transmission system. This high PAPR due to power amplifier nonlinearity, causes inter modulation and out-of-band radiation. High linearity indicates low efficiency and greater power dissipation, which should be least for use in portable devices [1]. Thus, it is highly desirable to improve PAPR performance of the signal.

\footnotetext{
Many techniques have been developed and used to overcome the effect of PAPR. These techniques are categorized as linear and non linear techniques. Some of them are Tone Injection, Clipping, Companding, Selective Mapping (SLM), Dummy sequence insertion (DSI) and Partial Transmit Sequence. In this paper a combination of PTS and DSI has been proposed.
}

\begin{abstract}
PTS and SLM have proved to deliver best PAPR reduction, at the expense of computational complexity [2]. PTS is distortionless but is very time-consuming, since it needs large number of computations [3]. DSI reduces the transmission efficiency of the signal. But in this technique by combining both DSI and PTS, the benefits of both have been utilized. The main issue of concern in DSI is the Transmission Efficiency which is inversely proportional to the number of subcarriers. And the trial and error procedure in Partial Transmit Sequence increases the computational complexity. More the number of iterations, greater will be the time consumption. When Mobile Wimax is considered a large number of data subcarriers will considerably increase the computational complexity. In this paper, by using the combination of DSI-PTS technique, the PAPR is improved, also the computational complexity is reduced, which means reduction in time consumption.
\end{abstract}

The number of sub blocks in PTS and the number of dummy subcarriers has been changed to analyze the effect on PAPR. By using DSI-PTS technique, the PAPR is reduced as compared to the conventional PTS and reduction in the computational complexity is achieved. The simulations have been observed for Mobile Wimax standard by varying the number of sub blocks and dummy subcarriers. PAPR is prominently reduced by PTS technique, but the complexity of the system is observed to increase exponentially with the number of sub blocks [4]. Since DSI technique reduces the PAPR but does not increase the complexity issue, so the combination of the two techniques have been simulated to obtain a less complex method for reducing PAPR. DSI is a frequency domain method which is implemented prior to the time domain method i.e. PTS [5].

\section{OFDM System and Problem of PAPR 2.1 OFDM System.}

In OFDM system, N' symbols is formed. Each set of subcarrier modulates each symbol. 


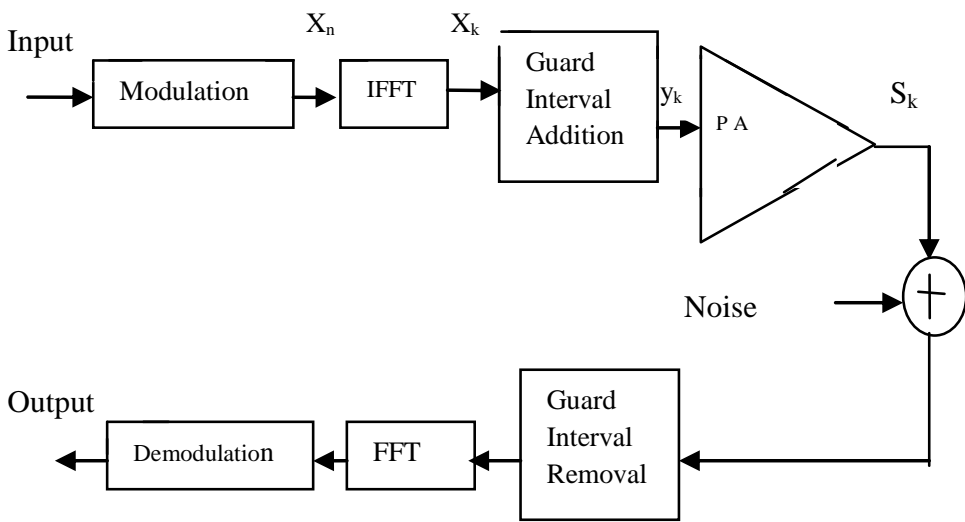

Figure 1: Block Diagram of OFDM System.

\section{2 . Issue of PAPR}

The sum of number of sub carriers of OFDM symbols has been compared with the single carrier system, where synthesis may lead to a high peak power. This high peak power is expressed as the ratio between the maximum power and its average power as

$$
\operatorname{PAPR}(d B)=10 \log _{10} \frac{\max \left\{\left|s_{n}\right|^{2}\right\}}{E\left\{\left|s_{n}\right|^{2}\right.}
$$

$S_{n}$ is the signal processed by IFFT, where $S_{n}$ is

$$
s_{n}=\frac{1}{\sqrt{N}} \sum_{k=0}^{n-1} X_{k} W_{N}^{K n}
$$

For $\mathrm{N}$ subchannels, the PAPR is $\mathrm{N}$ times the mean power which can be represented as:

$P A P R=10 \log _{10} N$

Where $\quad X=\left[X_{0, \ldots . .,} X_{N / 2, \ldots \ldots . .} X_{N-1}\right]^{T}$ and $\mathrm{F}$ is the oversampling factor.

Thus the computed PAPR of the F-time oversampled signal is expressed as

$$
\operatorname{PAPR}\left\{x_{n / F}\right\}=\frac{\max \left|x_{n / F}\right|^{2}}{E\left[\left|x_{n / F}\right|^{2}\right]}
$$

All material on each page should fit within a rectangle of $18 \mathrm{x}$ $23.5 \mathrm{~cm}(7 " \mathrm{x} 9.25 ")$, centered on the page, beginning $2.54 \mathrm{~cm}$ (1") from the top of the page and ending with $2.54 \mathrm{~cm}(1 ")$ from the bottom. The right and left margins should be $1.9 \mathrm{~cm}$ (.75"). The text should be in two $8.45 \mathrm{~cm}(3.33 ")$ columns with a $.83 \mathrm{~cm}(.33 ")$ gutter.

\section{PROPOSED SCHEME}

\subsection{DSI-PTS Technique}

The proposed work include two techniques; Dummy Subcarriers insertion followed by Partial Transmit Sequence generation. The dummy subcarriers are generated and then added to the data subcarriers, forming a new vector. Here the number of dummy subcarriers and data subcarriers is B and D respectively. The new vector can be represented as:

$$
S=\left[X_{k}, W_{b}^{\prime}\right]
$$

Here $b=1,2 \ldots B$ is the dummy signal vector and $\mathrm{D}$ is the data subcarrier.

After the optimum OFDM signal has been generated, the PAPR is compared for allowed threshold value in the comparator block, which is predefined. The OFDM signal is transmitted if the PAPR value is acceptable and within threshold limit. If the value lies beyond threshold limit, a dummy subcarrier is inserted again and again till the optimum value of PAPR is obtained. As shown in the Figure 2, first the DSI is performed and then PTS is performed.

To explain the concept of DSI-PTS scheme, consider B is the number of dummy subcarriers and IFFT length for simulation is 128 , since scalable Wimax has been considered where the number of data subcarriers is 72 and that of used null subcarriers is 40 [6]. Dummy subcarriers will be replaced with zeros in data at receiver end.

The main objective is to analyze an optimum signal with least Peak to Average Power Ratio. After the value of signal has been achieved to be optimum, the PAPR is compared with the threshold value. If the value is in the threshold limit, it is passed on to the next block, if the desired value is not obtained, the signal is transmitted back to DSI block for generation of Dummy Subcarriers again [7] until the required PAPR is obtained.

\subsection{Computational Complexity}

If the oversampling factor is $\mathrm{F}=2^{\mathrm{f}}$ and Subcarriers is $\mathrm{N}=2^{\mathrm{n}}$; the total complexity of addition and multiplications for conventional Partial Transmit Sequence and DSI-PTS technique and can be calculated as

$$
\begin{aligned}
& T_{C-P T S}=\frac{3 M N}{2} \log N+\frac{3 M N}{2} \\
& T_{D S I-P T S}=\frac{3 M N}{4} \log N+\frac{3 M N}{2}+L
\end{aligned}
$$

Where $\mathrm{M}$ is the number of subblocks and $\mathrm{N}$ is the total number of subcarriers.

The computational complexity reduction ratio CCRR is expressed as

CCRR $=\left(1-\frac{\text { Computational Complexity of C }- \text { PTS }}{\text { Computational Complexity of DSI }- \text { PTS }}\right) \times 100 \%$

Table 1. Comparison of CCRR for fixed and Mobile Wimax for subblocks $M=2$

\begin{tabular}{|c|l|l|}
\hline $\mathbf{M = 2}$ & $\begin{array}{l}\mathbf{N = 2 5 6} \\
\mathbf{L}=\mathbf{5 5}\end{array}$ & $\begin{array}{l}\mathbf{N}=\mathbf{5 1 2} \\
\mathbf{L}=\mathbf{9 2}\end{array}$ \\
\hline $\boldsymbol{T}_{\boldsymbol{C}-\text { PTS }}$ & 2617 & 5697 \\
\hline $\boldsymbol{T}_{\text {DSI-PTS }}$ & 1747 & 3708 \\
\hline
\end{tabular}




\section{CCRR}

$33.3 \%$

Table 2.

Comparison of CCRR for fixed and Mobile Wimax for subblocks $M=4$

\begin{tabular}{|c|l|l|}
\hline $\mathbf{M}=\mathbf{4}$ & $\begin{array}{l}\mathbf{N}=\mathbf{2 5 6} \\
\mathbf{L = 5 5}\end{array}$ & $\begin{array}{l}\mathbf{N}=\mathbf{5 1 2} \\
\mathbf{L}=\mathbf{9 2}\end{array}$ \\
\hline $\boldsymbol{T}_{\text {C-PTS }}$ & 5234 & 11358 \\
\hline $\boldsymbol{T}_{\text {DSI-PTS }}$ & 3440 & 7325 \\
\hline CCRR & $34.3 \%$ & $35.6 \%$ \\
\hline
\end{tabular}

Table 1and 2 demonstrate the CCRR of proposed DSI-PTS scheme as compared with Conventional PTS for fixed and mobile Wimax systems. The values of PTS sub blocks have been considered to be $\mathrm{M}=2$ and 4 .

The computational complexity of DSI-PTS can be reduced in terms of the number of subblock partition. For different values of $\mathrm{M}$, the complexity can be reduced significantly.

\subsection{Transmission Efficiency}

The OFDM signal faces no signal distortion after making use of C-PTS method, but when DSI is implemented with PTS, the transmission efficiency is expressed as

$T_{\eta}=\frac{K}{K+D} \times 100 \%$

Here number of subcarriers is defined by $\mathrm{K}$ and $\mathrm{D}$ represents Dummy Subcarriers. Since the value of D is replaced with zeros, so Transmission efficiency is theoretically hundred percent. As a result for increasing linearity of power amplifier, high peak signals can be transferred to power amplifier improving the PAPR performance [9-10]. An increase in efficiency of amplifiers and reduction in cost of system is achievable.

\section{SIMULATIONS AND RESULTS}

MATLAB simulation was performed on scalable i.e. mobile Wimax for 512 subcarriers of which maximum length of data subcarriers was 512 and dummy subcarriers were 92 . The oversampling factor was assumed to be 4 . Flipping technique is used for Dummy Sequence Insertion in the proposed method.

Figure 3 shows the CCDF plot of C-PTS and proposed DSIPTS methods. The number of dummy subcarriers for simulation was fixed at 92 and doesn't affect the transmission efficiency significantly [11]. An improvement of nearly 2 and

\section{CONCLUSION AND FUTURE SCOPE}

A combination of Dummy Subcarrier Insertion and Partial Transmit Sequence Scheme has been proposed in this paper. The computational complexity is lesser as compared to
$2.5 \mathrm{~dB}$ is obtained at $\mathrm{CCDF}=.01 \%$ at $\mathrm{M}=2$ and 4 respectively for dummy subcarriers 92 respectively.

The PAPR is reduced with the rise in the number of subblocks, but up to a certain extent. If the number is further increased from 4, the cost is complexity for improvement in PAPR performance.

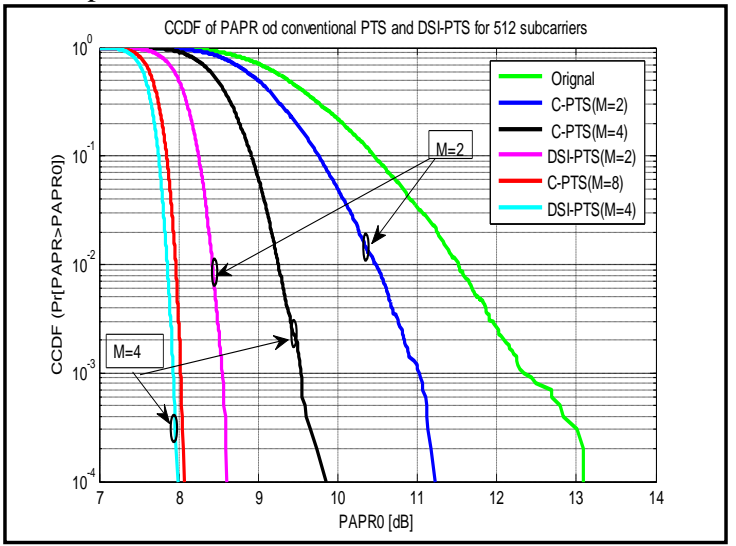

Figure 3: CCDF of the PAPR of conventional PTS and DSI-PTS technique when the number of DSI is 92 and the number of sub blocks is $M$ is varied for mobile Wimax.

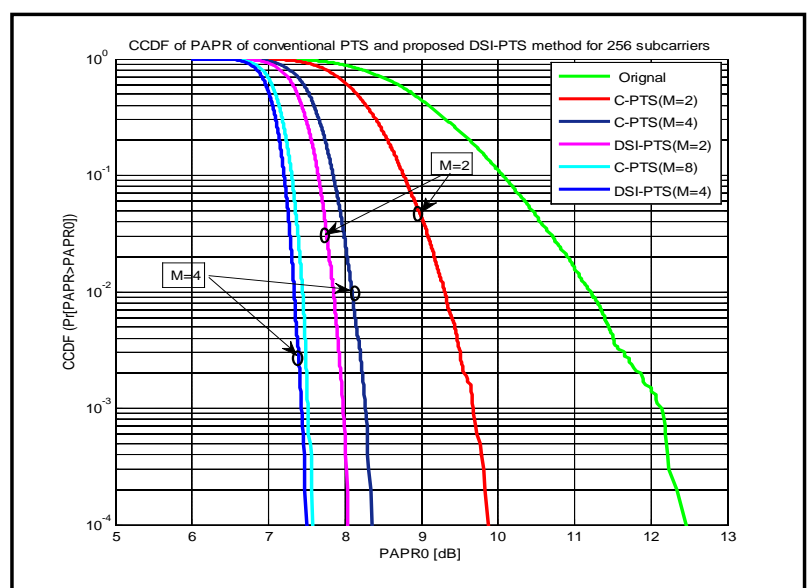

Figure 4: CCDF of the PAPR of conventional PTS and DSI-PTS technique when the number of DSI is 55 and the number of sub blocks are varied for fixed Wimax.

Figure 4 shows the simulation result for fixed Wimax for conventional PTS method and proposed method for fixed number of dummy subcarriers i.e. 55. The values have been compared for number of subblocks $=2$ and 4 respectively. For $\mathrm{CCDF}=0.01 \%$ as the number of subblocks increases, the PAPR reduces. The reduction has been achieved to a level of $1.5 \mathrm{~dB}$ and $2 \mathrm{dBs}$. The complexity increases as the number of subblocks increases. As compared to conventional PTS there is significant improvement in complexity because dummy subcarriers are included in data value which reduces the IFFT computations according to Table 1 and 2 .

conventional PTS technique. The simulation has been performed for 128 data subcarriers by using Matlab for different values of Dummy Subcarriers and the number of subblocks in PTS. By applying the proposed scheme, the 
number of IFFT computations are reduced which reduces the complexity of the system, and the PAPR performance is also improved. The application of the proposed technique will be useful in Wimax applications.

This work includes modification in terms of number of data subcarriers and the number of subcarriers to improve the crest factor. The simulation results show that the proposed technique performs as good as in Fixed Wimax environment. The PAPR improvement as compared to conventional Partial Transmit Sequence is achieved to be better with proposed method. The PAPR is improved by as much as $3 \mathrm{~dB}$ for scalable Wimax. The computational complexity reduction ratio calculated upto $36.8 \%$ for mobile Wimax. This indicates that the proposed technique can be used in mobile Wimax as well.

The QPSK modulation used in simulations may be replaced with other modulations such as QAM and the effect of modulation type can be examined.

\section{REFERENCES}

[1] B. Razavi, RF Microelectronics. Englewood Cliffs, NJ: Prentice Hall,1998.Ding, W. and Marchionini, G. 1997 A Study on Video Browsing Strategies. Technical Report. University of Maryland at College Park.

[2] Di-xiao Wu, "Selected Mapping and Partial Transmit Sequence Schemes to Reduce PAPR in OFDM Systems", IEEE International Conference on Image Analysis and Signal Processing, pp.1-5, October 2011. Tavel, P. 2007 Modeling and Simulation Design. AK Peters Ltd.

[3] L. J. Cimini and N. R. Sollenberger, "Peak-to-average power ratio reduction of an OFDM signal using partial transmit sequences," IEEE Communications. Letters., Vol. 4, No. 3, pp. 86-88., March. 2000.

[4] C. Tellambura, "Improved phase factor computation for the PAR reduction of an OFDM signal using PTS," IEEE
Commuication. Lettters, Vol. 5, No. 4, pp. 135-137, April 2001Brown, L. D., Hua, H., and Gao, C. 2003. A widget framework for augmented interaction in SCAPE.

[5] L. Yang, R. S. Chen, Y. M. Siu, and K. K. Soo, "PAPR reduction of an OFDM signal by use of PTS with low computational complexity," IEEE Transactions on Broadcasting, Vol. 52, No. 1, pp. 83-86, March 2006.

[6] Heung-Gyoon Ryu, Jae-Eun Lee and Jin-Soo Park, “ Dummy Sequence Insertion (DSI) for PAPR Reduction", IEEE Transactions on Consumer Electronics, Vol. 50, No. 1, pp. 89-94, January 2004.

[7] Tao Jiang, Weidong Xiang, Paul C. Richardson, Jinhua Guo, and Guangxi Zhu, "PAPR Reduction of OFDM Signals Using Partial Transmit Sequences With Low Computational Complexity", IEEE Transactions on Broadcasting, Vol. 53, No. 3, pp. 719-724, March 2007.

[8] Hadj Zerrouki, Mohamed Feham, "High Throughput of WiMAX MIMO-OFDM Including Adaptive Modulation and Coding", International Journal of Computer Science and Information Security, Vol. 7, No. 1, pp. 2547- 2552, January 2010.

[9] L. Yang, R. S. Chen, Y. M. Siu, and K. K. Soo, "PAPR Reduction of an OFDM Signal by Use of PTS With Low Computational Complexity", IEEE Transactions on Broadcasting, Vol. 52, No. 1, pp. 83-86, January 2006.

[10] Pooria Varahram, Wisam F. Al-Azzo, Borhanuddin Mohd Ali, "A Low Complexity Partial Transmit Sequence Scheme by Use of Dummy Signals for PAPR Reduction in OFDM Systems", IEEE Transactions on Consumer Electronics, Vol. 56, No. 4, pp. 2416-2420, April 2010.

[11] Haitao Xiong, Ping Wang, Zhiyong Bu, "An Efficient Peak-to-Average Power Ratio Reduction Algorithm for WiMAX System", IEEE Asia-Pacific Conference on Communications, pp. 1 - 5, May 2006. 